\title{
Comparison of enhanced recovery protocol with conventional care in patients undergoing minor gynecologic surgery
}

\author{
Gulseren Yilmaz ${ }^{1}$, Aysu Akca², Huseyin Kiyak², Ziya Salihoglu ${ }^{1}$ \\ ${ }^{1}$ Department of Anesthesiology and Reanimation, Faculty of Medicine, University of Health Sciences, Kanuni Sultan Suleyman Hospital, \\ Istanbul, Turkey \\ 2Department of Obstetrics and Gynecology, University of Health Sciences, Faculty of Medicine, Kanuni Sultan Suleyman Hospital, \\ Istanbul, Turkey
}

Videosurgery Miniinv 2020; 15 (1): 220-226

DOI: https://doi.org/10.5114/wiitm.2019.85464

\begin{abstract}
Introduction: Data regarding the role of the enhanced recovery after surgery (ERAS) protocol in improving postoperative outcomes and postoperative compliance in patients undergoing gynecological surgery, in particular, minor laparoscopic and hysteroscopic gynecological procedures, are limited.

Aim: To investigate the impact of the ERAS protocol on time to ambulation, length of stay (LOS), readmissions and postoperative complications in patients undergoing minor gynecological surgical procedures.

Material and methods: A total of 104 patients undergoing minor laparoscopic and hysteroscopic gynecological procedures were randomized to the ERAS protocol or conventional care. Time to defecation, ambulation, and solid food intake, bleeding and LOS were recorded for each patient.

Results: The amount of intravenous fluid administered in the perioperative $(p<0.001)$ and postoperative period $(p<0.001)$ was significantly higher in the conventional care group than in the ERAS group. In addition, time to first defecation $(p<0.001)$, time to eating solid food $(p<0.001)$, and time to ambulation $(p=0.008)$ were shorter in the ERAS group compared to the conventional care group. Length of stay was also significantly shorter in the ERAS group than in the conventional care group $(p<0.001)$.

Conclusions: Implementation of ERAS protocols provides shorter LOS, less fluid intake, early return of bowel function and early mobilization without an increase in complication rate in women undergoing minor laparoscopic or hysteroscopic gynecologic surgery.
\end{abstract}

Key words: gynecologic surgery, enhanced recovery after surgery, enhanced recovery, laparoscopy, hysteroscopy.

\section{Introduction}

The historical perioperative management concept named as 'wait and see' was a static and passive process where the physician reacted upon the presence of several postoperative complications. However, a paradigm shift towards a more active preand postoperative management strategy occurred following the implementation of enhanced recovery techniques which was initially proved successful in colorectal surgery [1].

Enhanced recovery after surgery (ERAS), or in other words the "fast-track" protocol, roughly purposes to improve patient satisfaction, reduce complications and shorten the hospital stay. Chronic diseases, nutrition, and any volume depletion are corrected prior to surgery and less invasive surgical techniques are used to this end [2]. In addition, ade-

\section{Address for correspondence}

Gulseren Yilmaz MD, Department of Anesthesiology and Reanimation, Faculty of Medicine, University of Health Sciences,

Kanuni Sultan Suleyman Hospital, Istanbul, Turkey, e-mail: drgulseren83@gmail.com 
quate pain and temperature control, and goal-directed fluid administration are required intraoperatively. In the postoperative period nutrition should be optimized, and early mobilization and early removal of the catheters and drains are carried out to achieve the goals of ERAS [3].

The impact of ERAS protocols in decreasing length of stay (LOS), reducing postoperative pain, improving early ambulation and decreasing the rate of potentially serious medical complications has been studied in patients undergoing colorectal, urologic, gastric and pancreatic surgery previously [4-7]. However, data regarding the role of ERAS protocol in improving postoperative outcomes and postoperative compliance in patients undergoing gynecological surgery, in particular, minor laparoscopic and hysteroscopic gynecological procedures, are limited.

\section{Aim}

The present study aimed to investigate the impact of the ERAS protocol on time to ambulation, LOS, readmissions and postoperative complications in patients undergoing minor gynecological surgical procedures.

\section{Material and methods}

Out of the 112 eligible patients undergoing minor laparoscopic and hysteroscopic gynecological procedures between March 2017 and April 20128 in our institute, 104 patients not meeting exclusion criteria were enrolled in this prospective, randomized and controlled study. Informed consent has been obtained from all patients. The study protocol was approved by the Institutional Ethics Committee (KAEK/2017.3.3) and registered with the National Ministry of Health, Health Sciences University (Turkey, Istanbul) and the study was performed in accordance with the most recent version of the Helsinki Declaration. Emergent surgery, admission to intensive care unit following surgery, preoperative sepsis, advanced liver and kidney disease were the exclusion criteria. Using random allocation software (www.randomization.com), patients were randomly allocated to one of the following study groups: the ERAS group or the conventional care group which consisted of age-matched control patients who did not receive ERAS interventions (Figure 1). A randomization envelope was opened at the time of admission to identify the study group of the index patient.
In the conventional group, patients were admitted the day before the surgery. All patients received a urinary catheter and prophylactic antimicrobial agent (cefoperazone $1000 \mathrm{mg}$ ) in the operation room just before the procedure and subsequently underwent general anesthesia. Parenteral intravenous fluid supplementation of $100 \mathrm{ml} / \mathrm{h}$ was performed in all patients and was removed as soon as the patient was able to eat and drink. Postoperative analgesia was achieved using oral paracetamol $1000 \mathrm{mg}$ and diclofenac $50 \mathrm{mg}$ three times daily.

ERAS group subjects were admitted on the day of the surgery. A standardized ERAS protocol was applied to the ERAS group based on the latest guidelines [8]. Smoking and alcohol consumption was stopped 4 weeks before the surgery. Preoperative anemia was corrected with intravenous iron supplementation and hematology counseling was performed in advanced cases. Prolonged fasting, bowel preparation, and premedication were avoided in this group. Clear fluids were allowed up to $2 \mathrm{~h}$ and solids rich in carbohydrate up to $6 \mathrm{~h}$ prior to induction of anesthesia. Routine antibiotic prophylaxis (cefoperazone $1000 \mathrm{mg} 60 \mathrm{~min}$ before the skin incision) and selective thromboembolism prophylaxis using low-molecular-weight heparin were given preoperatively. Warmed up intravenous fluids were administered to maintain normothermia intraoperatively. They received short-acting anesthetic agents during of midthoracic, epidural anesthesia/analgesia intraoperatively and postoperatively [8]. Volume and salt overload and drain usage were avoided to the utmost. Intravenous paracetamol was administered for postoperative analgesia before completion of the surgical procedure. Postoperative nausea was treated with ondansetron as the first choice and metoclopramide if needed. Nasogastric tube placement was avoided and catheters were removed as soon

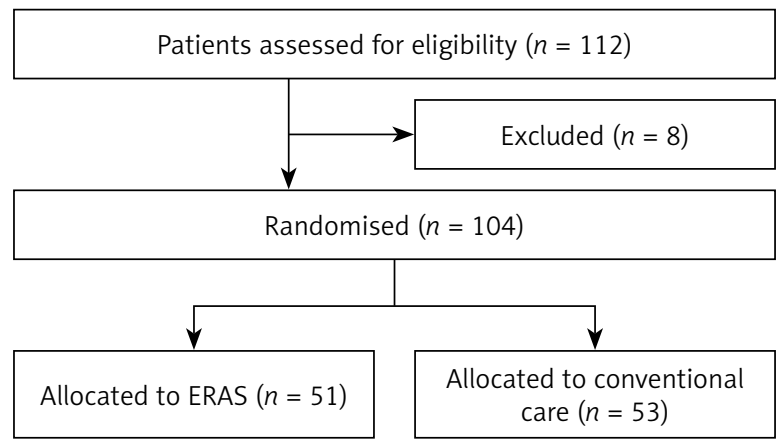

Figure 1. CONSORT Flow diagram 
as possible. Nonopioid oral analgesics and NSAIDs were used for postoperative pain medication. Oral intake was allowed at an early period and mobilization was induced as early as possible [9].

The two groups were compared in terms of complications and postoperative compliance including time to first flatus, defecation, ambulation, and solid food intake, bleeding and hospital stay.

\section{Statistical analysis}

Statistical analyses were carried out using SPSS for Windows, version 17 (SPSS, Chicago, IL, USA). Power calculations based on our pilot study with 40 patients to detect a $20 \%$ significant difference in time to ambulation between the groups indicated that at least 46 patients were needed in each group (G power 3, Dusseldorf University). Normal distribution of the variables was studied with the Kolmogorov-Smirnov test. Continuous variables are presented as the mean \pm standard deviation and categorical variables as a percentage. Student's $t$-test was used for parametric comparisons and the Mann-Whitney $U$ test was used for non-parametric comparisons.
The $\chi^{2}$ test was used for univariate analysis of the categorical variables. Two-sided $p \leq 0.05$ was interpreted as statistically significant.

\section{Results}

The conventional care group consisted of 53 patients (mean age: $39.1 \pm 10$ years) and the ERAS group consisted of 51 patients (mean age: $37.2 \pm 10$ years). The two groups were similar with respect to age $(p=0.334)$, body mass index $(p=0.355)$, ASA score $(p=0.128)$, preoperative diagnosis, and the surgical procedure performed. Adnexal mass was the most common preoperative diagnosis and salpingo-oophorectomy was the most frequent surgical procedure for both groups (Table I).

The amount of intravenous fluid administered in the perioperative $(1643 \pm 202 \mathrm{ml}$ vs. $1134 \pm 153 \mathrm{ml}$, $p<0.001)$ and postoperative period $(1701 \pm 329 \mathrm{ml}$ vs. $1003 \pm 217 \mathrm{ml}, p<0.001)$ was significantly higher in the conventional care group than in the ERAS group. In addition, time to first flatus $(11.4 \pm 2.2 \mathrm{~h}$ vs. $14.8 \pm 2.2 \mathrm{~h}, p<0.001)$, time to first defecation $(22.8$ \pm 1.5 h vs. $26.4 \pm 3.4$ h, $p<0.001)$, time to eating sol-

Table I. Preoperative characteristics of the study groups

\begin{tabular}{|c|c|c|c|}
\hline Parameter & $\begin{array}{c}\text { ERAS } \\
(n=51)\end{array}$ & $\begin{array}{c}\text { Conventional care } \\
\quad(n=53)\end{array}$ & $P$-value \\
\hline Age [years] & $37.2 \pm 10$ & $39.1 \pm 10$ & 0.334 \\
\hline $\mathrm{BMI}\left[\mathrm{kg} / \mathrm{m}^{2}\right]$ & $27.1 \pm 6.6$ & $26.1 \pm 3.8$ & 0.355 \\
\hline ASA score & $1.98 \pm 0.25$ & $1.90 \pm 0.29$ & 0.128 \\
\hline \multicolumn{4}{|l|}{ Diagnosis, $n(\%)$ : } \\
\hline Adnexal mass & $14(27.4)$ & $16(30.1)$ & 0.758 \\
\hline Uterine myoma & $10(19.6)$ & $12(22.6)$ & 0.705 \\
\hline Endometrial polyp & $6(11.7)$ & $10(18.8)$ & 0.218 \\
\hline Ovarian cyst & $8(15.6)$ & $6(7.5)$ & 0.514 \\
\hline Other & $13(25.4)$ & $9(16.9)$ & 0.288 \\
\hline \multicolumn{4}{|l|}{ Surgical procedure, $n(\%)$ : } \\
\hline Salpingo-oophorectomy & $16(31.3)$ & $18(33.9)$ & 0.648 \\
\hline Hysteroscopic myomectomy & $11(27.4)$ & $14(26.4)$ & 0.563 \\
\hline Endometrial polyp resection & $6(20.4)$ & $10(18.8)$ & 0.218 \\
\hline Ovarian cystectomy & $8(15.6)$ & $6(7.5)$ & 0.514 \\
\hline Fallopian tube ligation & 10 (19.6) & $5(9.4)$ & 0.140 \\
\hline Perioperative intravenous fluids [ml] & $1134 \pm 153$ & $1643 \pm 202$ & $<0.001$ \\
\hline
\end{tabular}

Data are presented as mean \pm standard deviation. BMI - body mass index, ERAS - enhanced recovery after surgery. 
id food (13.9 \pm 4.2 h vs. $18.7 \pm 5.1$ h, $p<0.001)$, and time to ambulation $(20.3 \pm 1.5$ h vs. $23.9 \pm 3.3 \mathrm{~h}, p=$ 0.008 , Table II) were shorter in the ERAS group compared to the conventional care group. Moreover, hospital stay was also significantly shorter in the ERAS group when compared with the conventional care group ( $1.13 \pm 0.4$ days vs. $1.64 \pm 0.5$ days, $p<0.001$ ).

The two groups were similar concerning the postoperative complications including surgical site infections ( $7.8 \%$ vs. $13.2 \%, p=0.374)$, development of cardiovascular complications (1.9\% vs. $3.7 \%, p=$ $0.581)$, non-specific abdominal pain ( $9.8 \%$ vs. $7.5 \%$, $p=0.801$ ), and sub-ileus ( $0 \%$ vs. $1.8 \%, p=0.324$ ). Blood loss (162 $\pm 44 \mathrm{ml}$ vs. $171 \pm 58 \mathrm{ml}, p=0.345$ ), overall complication rate ( $20 \%$ vs. $32 \%, p=0.213)$ and readmission rate ( $4 \%$ vs. $6 \%, p=0.679)$ were also similar in the two groups (Table III).

\section{Discussion}

The present study aimed to investigate the impact of ERAS protocols on postoperative recovery in patients undergoing minor laparoscopic or hysteroscopic gynecologic surgery and demonstrated that compared to conventional care, the implementation of the ERAS protocol leads to significantly short- er LOS, early mobilization and reduced fluid intake without an increase in complication rate.

One of the most promising improvements in surgery for perioperative care in recent years is the introduction of structured multimodal treatment programs which are also called ERAS protocols. Faster recovery and shorter hospital stay obtained with implementation of ERAS protocols were shown to be associated with improved patient appreciation in a recent study which enrolled patients undergoing elective laparoscopic procedures [10]. Furthermore, these protocols have not only been shown to reduce recovery times but also decrease the complication rates by as much as $50 \%$, and the complication rate and LOS have been shown to improve in accordance with the increasing compliance rates $[11,12]$. The international ERAS Study group, therefore, has recently published guidelines for various surgical procedures to establish a standardized way to apply ERAS protocols in the perioperative period $[8,9]$.

The principle underlying the ERAS programs is to reduce the metabolic stress caused by surgery combined with use of treatments that support reversion of functions that postpone the recovery in traditional care and consequently allow the patient to recov-

Table II. Postoperative outcomes

\begin{tabular}{|lccc|}
\hline Parameter & $\begin{array}{c}\text { ERAS } \\
(n=51)\end{array}$ & $\begin{array}{c}\text { Conventional care } \\
(n=53)\end{array}$ & $P$-value \\
\hline Time to first flatus $[\mathrm{h}]$ & $11.4 \pm 2.2$ & $14.8 \pm 2.2$ & $<0.001$ \\
\hline Time to first defecation $[\mathrm{h}]$ & $22.8 \pm 1.5$ & $26.4 \pm 3.4$ & $<0.001$ \\
\hline Time to ambulation $[\mathrm{h}]$ & $20.3 \pm 1.5$ & $23.9 \pm 3.3$ & 0.008 \\
\hline Time to eating solid food [h] & $13.9 \pm 4.2$ & $18.7 \pm 5.1$ & $<0.001$ \\
\hline Postoperative intravenous fluids [ml] & $1003 \pm 217$ & $1701 \pm 329$ & $<0.001$ \\
\hline Postoperative hospital stay [days] & $1.13 \pm 0.4$ & $1.64 \pm 0.5$ & $<0.001$ \\
\hline
\end{tabular}

Data are presented as mean \pm standard deviation.

Table III. Comparison of the complication and readmission rates between the two groups

\begin{tabular}{|lccc|}
\hline Parameter & $\begin{array}{c}\text { ERAS } \\
(n=51)\end{array}$ & $\begin{array}{c}\text { Conventional care } \\
(n=53)\end{array}$ & $P$-value \\
\hline Total complications, $n(\%)$ & $10(19)$ & $16(30.1)$ & 0.213 \\
\hline Blood loss [ml] & $162 \pm 44$ & $171 \pm 58$ & 0.345 \\
\hline Transfusion requirement, $n(\%)$ & $1(0.19)$ & $4(7.5)$ & 0.183 \\
\hline Readmission, $n(\%)$ & $2(3.9)$ & $3(5.6)$ & 0.679 \\
\hline
\end{tabular}

Data are presented as mean \pm standard deviation. 
er faster. In brief, due to the insulin resistance and the catabolic state caused by the stress of surgery, all parts of metabolism are confused, further leading to the development of complications and delay in recovery [13]. By avoiding the prolonged starvation periods and supplementation of a carbohydrate-rich diet, ERAS interventions improve insulin sensitivity and thus reduce the complications developing as a result of the surgery stress [14].

Another key factor in improving postoperative outcomes achieved by ERAS protocols is to maintain fluid balance. Administration of $0.9 \%$ saline as the crystalloid to avoid hypotension during and after surgery is a longstanding traditional attitude which challenges the results of the novel studies indicating that non-selective fluid overload not only is unnecessary but also might be harmful in the majority of patients, since this fluid is preserved in the body for a longer time than balanced salt solutions [15]. Studies have shown that fluid overload might also postpone the return of bowel function and, in contrast to volume overload, fluid restriction reduces the postoperative complication rate [16]. Fluid loads exceeding $3000 \mathrm{ml}$ has been found in association with increased complications for patients undergoing colonic resections [12]. In addition to aforementioned measures, avoidance of preoperative sedatives, long-acting anesthetics and minimizing the use of opioids will prevent the development of postoperative nausea and help to initiate the oral intake within hours following the operation [17]. The combination of these measures will provide a synergistic effect in the outcome expected from ERAS protocols. ERAS principles proved successful in decreasing mortality even in the long-term period in an observational study including 4500 patients undergoing hip and knee replacement [18].

In patients undergoing colorectal, urologic, gastric and pancreatic surgery substantial patient and healthcare benefits were acquired with the implementation of ERAS protocols by decreasing LOS, complication rate, and lowering hospital costs [1922]. ERAS principles have also long been used in women undergoing major gynecologic surgery. Results derived from an observational study conducted by Wijk et al. revealed that implementation of ERAS protocol leads to a significant increase in the number of patients discharged within 2 days after the surgery [23]. Similarly, Mukhopadhyay reported that in patients undergoing open hysterectomies,
LOS decreased from 5 days to 3 days with the implementation of ERAS protocols [24]. Ottensen et al. showed that median LOS following vaginal surgery performed for utero-vaginal prolapse was also significantly lower in patients receiving ERAS protocols compared to those receiving conventional care [25]. The amount of intraoperative fluid intake and total fluid intake was also shown to be decreased with the implementation of the ERAS protocol in women operated on for gynecologic cancer [26].

In addition, the return of bowel function and early ambulation, which are closely related to early discharge, are significantly improved when ERAS protocols are used. Hansen et al. observed in a double-blind, randomized controlled trial of patients undergoing abdominal hysterectomy that time to defecation was $24 \mathrm{~h}$ sooner in the ERAS group compared to controls [27]. The impact of implementing ERAS protocols on ambulation in abdominal hysterectomy and utero-vaginal prolapse surgery was assessed in two studies where ERAS was found to be significantly associated with early ambulation and consequently early discharge $[25,27]$. Furthermore, studies investigating the ERAS protocol in gynecologic surgery reported that the benefits were acquired without an increase in complication rates irrespective of the surgical approach [3].

Although extensive research is available concerning the benefits of ERAS in major gynecologic surgery, data demonstrating its effectiveness in minor gynecologic procedures are less clear. Therefore, our results demonstrating the advantages of the ERAS protocol for LOS, fluid intake, early defecation and early ambulation in patients undergoing minor gynecologic procedures indicate that the implementation of these measures might be useful even in less invasive gynecologic procedures.

The present study also has some limitations to be discussed. A blinded study protocol was not carried due to the extensive multidisciplinary nature of the ERAS protocol; thus, physicians and the healthcare workers were not blinded to the study protocol. Another limitation of the present study is that the complication rates in our study were similar in ERAS and conventional care groups, in contrast to previous reports revealing fewer complications with the implementation of ERAS. As we enrolled patients undergoing minor gynecologic procedures, this kind of surgery is already less complicated and the complication rates are lower as well. Larger sample size 
would probably provide a more comprehensive comparison of the ERAS protocol and conventional care concerning the complication rate in this patient population.

\section{Conclusions}

Implementation of ERAS protocols provides shorter LOS, less fluid intake, early return of bowel function and early mobilization without an increase in complication rate in women undergoing minor laparoscopic or hysteroscopic gynecologic surgery. We suggest that developing an individual approach for each institute to adequately implement ERAS protocols in accordance with the guidelines published by the ERAS Study group might improve patient and healthcare outcomes not only in major surgery but also in minor gynecologic procedures.

\section{Conflict of interest}

The authors declare no conflict of interest.

\section{References}

1. Kehlet H, Dahl JB. Anaesthesia, surgery, and challenges in postoperative recovery. Lancet 2003; 362: 1921-8.

2. Yilmaz G, Akca A, Aydin N. Enhanced recovery after surgery (ERAS) versus conventional postoperative care in patients undergoing abdominal hysterectomies. Ginekol Pol 2018; 89: 351-6.

3. Scheib SA, Thomassee M, Kenner JL. Enhanced recovery after surgery in gynecology: a review of the literature. J Minim Invasive Gynecol 2019; 26: 327-43.

4. Mortensen K, Nilsson M, Slim K, et al. Consensus guidelines for enhanced recovery after gastrectomy: Enhanced Recovery After Surgery (ERAS(R)) Society recommendations. Br J Surg 2014; 101: 1209-29.

5. Cerantola Y, Valerio M, Persson B, et al. Guidelines for perioperative care after radical cystectomy for bladder cancer: Enhanced Recovery After Surgery (ERAS((R))) society recommendations. Clin Nutr 2013; 32: 879-87.

6. Lassen K, Coolsen MM, Slim K, et al. Guidelines for perioperative care for pancreaticoduodenectomy: Enhanced Recovery After Surgery (ERAS(R)) Society recommendations. Clin Nutr 2012; 31: 817-30.

7. Greco M, Capretti G, Beretta L, et al. Enhanced recovery pro gram in colorectal surgery: a meta-analysis of randomized controlled trials. World J Surg 2014; 38: 1531-41.

8. Nelson G, Altman AD, Nick A, et al. Guidelines for pre- and in tra-operative care in gynecologic/oncology surgery: Enhanced Recovery After Surgery (ERAS(R)) Society recommendations--Part I. Gynecol Oncol 2016; 140: 313-22.

9. Nelson G, Altman AD, Nick A, et al. Guidelines for postoperative care in gynecologic/oncology surgery: Enhanced Recovery
After Surgery (ERAS(R)) Society recommendations--Part II. Gynecol Oncol 2016; 140: 323-32

10. Zychowicz A, Pisarska M, Laskawska A, et al. Patients' opinions on enhanced recovery after surgery perioperative care principles: a questionnaire study. Videosurgery Miniinv 2019; 14: 27-37.

11. Gatt M, Khan S, MacFie J. In response to: Varadhan KK, Neal KR, Dejong $\mathrm{CH}$, Fearon KC, Ljungqvist O, Lobo DN. The enhanced recovery after surgery (ERAS) pathway for patients undergoing major elective open colorectal surgery: a meta-analysis of randomized controlled trials. Clin Nutr 29 (2010) 434-40. Clin Nutr 2010; 29: 689-90.

12. Gustafsson UO, Hausel J, Thorell A, et al. Adherence to the enhanced recovery after surgery protocol and outcomes after colorectal cancer surgery. Arch Surg 2011; 146: 571-7.

13. Ljungqvist O, Jonathan E. Rhoads lecture 2011: insulin resistance and enhanced recovery after surgery. JPEN J Parenter Enteral Nutr 2012; 36: 389-98.

14. Ljungqvist O. Modulating postoperative insulin resistance by preoperative carbohydrate loading. Best Pract Res Clin Anaesthesiol 2009; 23: 401-9.

15. Varadhan KK, Lobo DN. A meta-analysis of randomised controlled trials of intravenous fluid therapy in major elective open abdominal surgery: getting the balance right. Proc Nutr Soc 2010; 69: 488-98.

16. Brandstrup B, Tonnesen H, Beier-Holgersen R, et al. Effects of intravenous fluid restriction on postoperative complications: comparison of two perioperative fluid regimens: a randomized assessor-blinded multicenter trial. Ann Surg 2003; 238: 641-8.

17. Ljungqvist O. ERAS: enhanced recovery after surgery: moving evidence-based perioperative care to practice. JPEN J Parenter Enteral Nutr 2014; 38: 559-66.

18. Savaridas T, Serrano-Pedraza I, Khan SK, et al. Reduced medium-term mortality following primary total hip and knee arthroplasty with an enhanced recovery program. A study of 4,500 consecutive procedures. Acta Orthop 2013; 84: 40-3.

19. Adamina M, Kehlet $\mathrm{H}$, Tomlinson $\mathrm{GA}$, et al. Enhanced recovery pathways optimize health outcomes and resource utilization: a meta-analysis of randomized controlled trials in colorectal surgery. Surgery 2011; 149: 830-40.

20. Lin T, Li K, Liu H, et al. Enhanced recovery after surgery for radical cystectomy with ileal urinary diversion: a multi-institutional, randomized, controlled trial from the Chinese bladder cancer consortium. World J Urol 2018; 36: 41-50.

21. Ding J, Sun B, Song P, et al. The application of enhanced recovery after surgery (ERAS)/fast-track surgery in gastrectomy for gastric cancer: a systematic review and meta-analysis. Oncotarget 2017; 8: 75699-711.

22. Perinel J, Adham M. ERAS and pancreatic surgery: a review. Updates Surg 2016; 68: 253-5.

23. Wijk L, Franzen K, Ljungqvist O, Nilsson K. Implementing a structured Enhanced Recovery After Surgery (ERAS) protocol reduces length of stay after abdominal hysterectomy. Acta Obstet Gynecol Scand 2014; 93: 749-56.

24. Mukhopadhyay D. Enhanced recovery programme in gynaecology: outcomes of a hysterectomy care pathway. BMJ Qual Improv Rep 2015; 4: pii: u206142.w2524. 
25. Ottesen M, Sorensen M, Rasmussen $Y$, et al. Fast track vaginal surgery. Acta Obstet Gynecol Scand 2002; 81: 138-46.

26. Mendivil AA, Busch JR, Richards DC, et al. The Impact of an enhanced recovery after surgery program on patients treated for gynecologic cancer in the community hospital setting. Int J Gynecol Cancer 2018; 28: 581-5.

27. Hansen CT, Sorensen M, Moller C, et al. Effect of laxatives on gastrointestinal functional recovery in fast-track hysterectomy: a double-blind, placebo-controlled randomized study. Am J Obstet Gynecol 2007; 196: 311.e311-7.

Received: 20.03.2019, accepted: 29.04.2019. 